A Comparison of Multivariable Decentralized Control Strategies for Robust Humanoid Walking

Dallali, H. and Medrano-Cerda, G.A. and Brown, M. 2010

Manchester Institute for Mathematical Sciences

School of Mathematics

The University of Manchester

\footnotetext{
Reports available from:

http://eprints.maths.manchester.ac.uk/

And by contacting: The MIMS Secretary

School of Mathematics

The University of Manchester

Manchester, M13 9PL, UK
} 


\title{
A Comparison of Multivariable \& Decentralized Control Strategies for Robust Humanoid Walking *
}

\author{
H. Dallali, ${ }^{*}$ G.A. Medrano-Cerda, ${ }^{* *}$ M. Brown ${ }^{* * *}$ \\ * Centre for Interdisciplinary Computational and Dynamical Analysis \\ (CICADA), School of Mathematics, The University of Manchester, \\ M13 9PL, UK (e-mail: houman.dallali@postgrad.manchester.ac.uk). \\ ** Italian Institute of Technology (IIT), Via Morego, 3016163 Genova, \\ Italy (e-mail: gmedranocerda@gmail.com) \\ *** Control Systems Group, School of Electrical and Electronic \\ Engineering, The University of Manchester, M13 9PL, UK, (e-mail: \\ martin.brown@manchester.ac.uk)
}

\begin{abstract}
Bipedal walking is one of the most interesting control problems in humanoids research. Walking is modelled as a hybrid system in the sense that it involves various phases such as single support phase, impacts with the ground (i.e. a state reset) and the double support phase. The control system has to provide good dynamic performance in these different modes to achieve fast walking speeds while guaranteeing its safe and robust operation. Most humanoids use local joint PID loops (decentralized) control systems while the robot is a multivariable system and walking involves significant interactions between the robot links. Hence in this paper a centralized LQR multivariable controller is designed for the robot and analyzed for its stability, robustness to noise and disturbances and dynamic performance. Then, an LQR based iterative algorithm is used to tune the local PID servos. A comparison between the two schemes is done, where it is shown that the multivariable LQR has better robustness and energy efficiency. Finally, both controllers are simulated using the linearized model of a 10 degree of freedom robot called "C-Cub".
\end{abstract}

Keywords: Linear multivariable control, Decentralized control, Humanoid locomotion, C-Cub.

\section{INTRODUCTION}

Control of humanoids' walking involve significant challenges due to the high Degrees of Freedom (DoF), the presence of under actuated DoF, the hybrid nature of the system switching back and forth between single support and double support and external disturbances such as ground impacts on rough terrain when walking at high speeds. As the humanoids are built to operate autonomously their safe interaction is vital. Therefore verifying the safe operation of humanoids is the first priority for any control system. The design of a control system for humanoid locomotion has been widely studied in the literature, but there has been little work on their stability and robustness proofs. ASIMO, HRP3, HRP4 and HUBO2 are among the most advanced humanoids and have demonstrated successful walking and running experiments at relatively high speeds. Kaneko et al. (2005) presented a stabilizer module that works with local PID servo controllers to stabilize various versions of HRP robots. Kaneko et al. (2009) presented the most recent version of the HRP robots "HRP-4C" that has the same control architecture. The decentralized PID control is widely used for humanoid robots and most of the

\footnotetext{
* This research has been done on the CICADA project which is financed by the EPSRC grant EP/E050441/1 and the University of Manchester.
}

sophistication is transferred to the higher level stabilizer and trajectory generation. This is due to the fact that PID control is relatively simple to implement and most humanoids are designed with a decentralized hardware architecture with limited internal communication speed. Therefore the low bandwidth puts a major challenge for real-time centralized controller implementation. In addition, these robots use the concept of Zero Moment Point (ZMP) which was introduced thirty years ago by Vukobratovic and recently reviewed by Vukobratovic and Borovac (2004). ZMP is defined as a point on the ground where the total moments generated by the ground reaction forces are zero. Moreover, ZMP (as a sufficient condition) fails to utilize the full dynamics of the robots and it also does not provide a balance recovery strategy once it has been violated. Therefore, considerable attention has been given to developing more robust walking control systems in the last decade. Capture points introduced by Pratt et al. (2006) and foot placement estimators by Wight, et al. (2008) are among the new methods proposed. However, it should be noted that use of such concepts for trajectory generation is tightly related to the underlying control system architecture. In fact, since a humanoid robot is a multibody system, a fundamental question arises on how to choose the right stabilizing feedback controller. In this paper, the performance and stability robustness properties of a multivariable feedback system is compared to a 
decentralized PID feedback controller. Namely, the closed loop tracking bandwidth, energy efficiency and robustness to noise and disturbance are studied.

In section 2 , the model of walking in single support and double support phases is presented. A state space formulation is also given for the single support model to include reference tracking by introducing integrators. In section 3 , the single support state space model is used for the centralized and decentralized controller designs. An iterative algorithm is used to derive the decentralized feedback gains from a multivariable LQR formulation. In section 4 , the relative stability measures and the corresponding state space disturbance and noise models are presented. In section 5 , a model of $\mathrm{C}_{-} \mathrm{Cub}^{1}$ developed by the Robotcub project (2010) is used to illustrate the numerical results in terms of regulation, bandwidth, disturbance and noise rejection. Finally, conclusions and future directions are presented in the last section.

\section{MECHANICAL MODEL}

In this section, the mechanical model of a humanoid robot and the state space formulation is presented. CCub has 70 DoF, however for initial walking tests a minimal 3D dynamic model with 10 DoF is considered. The $10 \mathrm{DoF}$ include $5 \mathrm{DoF}$ in each leg. The rotation about vertical axis and the torso is not considered at this stage. Further modelling and simulation details are provided by Medrano-Cerda, et al. (2010) and the C-Cub mechanical model that includes all the dynamic parameters can be downloaded from: http://www.cicada.manchester.ac.uk/research/icub/

\subsection{Nonlinear single support model}

The dynamic model of single support (swing phase) where one foot is in contact with the ground is considered. The model is derived in symbolic form using Robotran that is a general dynamic Matlab based multi-body modelling software. The C-Cub model is:

$$
M(q) \ddot{q}+c(q, \dot{q})=u
$$

where $q$ is the angular joint positions in relative coordinates, $\dot{q}$ is the angular velocity, $M(q)$ is the mass inertia matrix which is a function of the 10 joint angles and $c(q, \dot{q})$ is the combined vector of coriolis, centripetal and gravitational forces that is a function of joint angles and velocities. $u$ is a vector of torques applied to the links. The joint angles, velocities and accelerations are expressed in relative coordinates. These model can be linearized about any operating point for linear control system design.

\subsection{Nonlinear double support model}

The double support model of C-Cub (where both feet are in contact with the ground) is presented as a constrained form of the single support model. The constraints result from the geometry of the double support phase where the robot maintains both legs on the ground.

\footnotetext{
1 C-Cub is a child humanoid robot developed by the Italian Institute of Technology. Robotcub project (2010)
}

$$
\begin{aligned}
& M(q) \ddot{q}+c(q, \dot{q})=u+J_{c}(q) \lambda \\
& h(q)=0, \quad \dot{h}(q)=J_{c}(q) \dot{q}=0, \quad \ddot{h}(q)=J_{c}(\ddot{q})+\dot{J}_{c} \dot{q}=0
\end{aligned}
$$

where, $h(q)$ is the nonlinear equality constraint, $J_{c}(q)$ is the jacobian of the constraint and $\lambda$ is the lagrange multiplier vector that represents the constraint forcestorques.

\subsection{The linearized single support model}

C-Cub Equation (1) is be linearized around the upright position, i.e.

$$
M_{r} \ddot{q}+G_{r} \dot{q}+K_{r} q=u
$$

Equation (3) is used in this paper to design the single support and double support controllers. The model in compact format is represented as:

$$
\dot{\tilde{x}}=\tilde{A} \tilde{x}+\tilde{B} u, \quad \tilde{y}=C \tilde{x}
$$

where $\tilde{x}=(q, \dot{q})^{T}$ is the state of the system, $u$ is the input torque to the system, $C=\left[\begin{array}{ll}I_{n} & 0\end{array}\right], I_{n}$ is an identity matrix of dimension $n$ and $n$ is the number of joints in the robot. Equation (4) represents the open chain model of the robot in single support which is fully controllable.

A control system must be designed that can track desired reference trajectories in face of modelling errors and disturbances. This is addressed by introducing integral action in the model. Let,

$$
\dot{z}=r-\tilde{y}=r-C \tilde{x}
$$

where $\tilde{y}$ is the measured position vector given in Equation (4) and $r$ is the reference input vector. The continuous time tracking system is described by:

$$
\dot{x}=A x+B u+B_{r} r, \quad y=C_{c l} x+D_{r} r
$$

where $x=\left[\begin{array}{c}\tilde{x} \\ z\end{array}\right], A=\left[\begin{array}{cc}\tilde{A} & 0 \\ -C & 0\end{array}\right], B=\left[\begin{array}{c}\tilde{B} \\ 0\end{array}\right], B_{r}=$ $\left[\begin{array}{c}\tilde{B} G_{f f} \\ I_{n}\end{array}\right], y=\left[\begin{array}{l}x \\ u\end{array}\right], C_{c l}=\left[\begin{array}{c}I_{p} \\ -K\end{array}\right], D_{c l}=\left[\begin{array}{c}0 \\ G_{f f}\end{array}\right], K$ is the state feedback gain and $G_{f f}$ is a feed forward gain that is defined in the next section. In addition, $I_{p}$ is an identity matrix of dimension $p$ where $p=3 n$. In the next section, the synthesis process for the centralized LQR and the decentralized PID feedback controllers is presented.

\section{CONTROL DESIGN}

In this section, an LQR feedback control is presented for stabilization and control of walking. Then, an iterative algorithm is used to design a stabilizing decentralized PID controller based on the LQR feedback controller. In this paper the negative feedback convention is used.

\subsection{Centralized LQR feedback controller design}

In order to obtain the continuous time optimal feedback gain $K$, the state feedback law $u=-K x$ must minimize the cost function $J$ subject to dynamics of the single support model.

$$
\min \quad J=\int_{0}^{\infty}\left(x^{T} Q x+u^{T} R u\right) d t
$$


subject to $\quad \dot{x}=A x+B u$

where $\mathrm{Q}$ is a positive semi-definite matrix and $\mathrm{R}$ is a positive definite matrix. It is well-known that the state feedback gain $K$ is the solution to the following algebraic Riccati equation:

$$
A^{T} P+P A-P B R^{-1} B^{T} P+Q=0
$$

solving the Riccati equation above for $P$ will result in the optimal state feedback gain:

$$
K=R^{-1} B^{T} P
$$

Hence the closed loop system can be formulated by:

$$
\dot{x}=A_{c l} x+B_{r} r
$$

where $A_{c l}=A-B K, B_{r}=\left[\begin{array}{ll}B G_{f f} & I_{n}\end{array}\right]^{T}$ and $G_{f f}=$ $K\left[\begin{array}{ll}I_{n} & 0\end{array}\right]^{T} . G_{f f}$ is referred to as the feed-forward gain and it is derived from the first block of the feedback gain $K$ that corresponds to the joint positions. The optimal gain $K$ serves as the basis for design of the decentralized controller using an iterative scheme which is presented in the next section.

\subsection{Decentralized PID feedback controller design}

In this section, a gradient based iterative method presented by Silva and Erraz (2006) is briefly reviewed and used to derive the decentralized feedback gains based on the LQR solution.

Levine and Athans (1970) have shown that the optimal cost function can be approximated by taking its average over a linearly independent set of initial conditions $x(0)$, which is equivalent to assuming that $x(0)$ is a random variable uniformly distributed over the surface of a pdimensional unit sphere. Hence the expected value of the cost function (7) is:

$$
\hat{J}=\frac{1}{2 p} \int_{0}^{\infty} \operatorname{tr}\left(\Phi^{T}(t, 0)\left(Q+K^{T} R K\right) \Phi(t, 0)\right) d t
$$

where $\Phi(t, 0)=e^{(A-B K) t}$ is the transition matrix. In addition, if $V(K)$ is defined as:

$$
V(K)=\int_{0}^{\infty}\left(\Phi^{T}(t, 0)\left(Q+K^{T} R K\right) \Phi(t, 0)\right) d t
$$

then the averaged cost $\hat{J}$ is:

$$
\hat{J}=\frac{1}{2 p} \operatorname{tr}[V(K)]
$$

It is shown by Levine and Athans (1970) that if $A_{c l}$ is stable, $V(K)$ is solution to the following Lyapunov equation:

$$
V A_{c l}+A_{c l}^{T} V+Q+K^{T} R K=0
$$

Furthermore, an iterative scheme can be used to solve the optimal control problem using the following gradientbased approach:

$$
K_{i}=K_{i-1}+\alpha \frac{d}{d K} \operatorname{tr}\left[V\left(K_{i-1}\right)\right]
$$

where $\alpha$ is a fixed step size used to achieve convergence to the optimal value. The value of the gradient is given as:

$$
\frac{d}{d K} \operatorname{tr}[V(K)]=2\left(-R K+B^{T} V(K)\right) W
$$

where $W$ is the solution to the following Lyapunov equation:

$$
A_{c l} W+W A_{c l}^{T}+I_{p}=0
$$

In design of decentralized controllers the actual optimal control solution has to be modified to cater for the sparse matrix $K$ that will be used by the local controllers. Silva and Erraz (2006) proposed the following updating policy:

$$
K_{i}=K_{i-1}+\alpha\left(\frac{d}{d K} \operatorname{tr}\left[V\left(K_{i-1}\right)\right] \cdot * E_{a}\right)
$$

where the operator ".* " denotes the termwise matrix multiplication as used in Matlab. The matrix $E_{a}$ is defined as:

$$
E_{a}=\left[\begin{array}{lll}
I_{n} & I_{n} & I_{n}
\end{array}\right]
$$

The algorithm presented above maintains the structure of the decentralized controller but the stability of the resulting gains may be lost. In such cases a stability check have to be performed and the non-stabilizing gains has to be discarded. Furthermore, to use this scheme the centralized LQR gain $K$ is used to derive the initial feedback gain $K_{0}=K$. * $E_{a}$, where $K_{0}$ has to be a stabilizing feedback gain for the multivariable system. It should be noted that the algorithm presented is a solution to the problem of decentralized PID design, but more general constrained gradient decent algorithms can be used to design the decentralized controllers using the available numerical optimisation tools.

Remark 1: A similar scheme can be used when the actuator dynamics is considered. The result of the decentralized controller would provide a PID on the motor side and a $\mathrm{PD}$ on the link side if the integrators are closed on the motor side and actuators are considered to be electric motors which is the case for C-Cub. In the next section some measures of stability and robustness analysis for multi-variable systems are presented for comparing the two centralized and decentralized control schemes.

\section{RELATIVE STABILITY AND ROBUSTNESS ANALYSIS}

The designed control system must tolerate uncertainties, disturbances and noise that are inherently present in any physical system. These uncertainties are caused by approximate models that represent the robot. In addition in the environment the robot is subject to external disturbances such as imperfect ground conditions, external forces and sensor noise, etc. Therefore, it is of interest to investigate the robust stability and performance of the control system under these circumstances. In this section, the state space models for the disturbance and measurement noise are presented. The frequency response of these models are used in the next section to study the disturbance and noise rejection of the feedback system.

\subsection{Disturbance and noise models}

In order to study the effects of both the disturbances on the plant output and the noise on the output measurement, the corresponding models are formulated in state space form. The disturbance and noise models are given by: 


$$
\begin{aligned}
& \dot{x}=A_{c l} x+B_{d} d \\
& y=C_{c l} x+D_{d} d \\
& \dot{x}=A_{c l} x+B_{n} m \\
& y=C_{c l} x+D_{n} m
\end{aligned}
$$

where $B_{d}=-B K-\left[\begin{array}{cc}0 & 0 \\ I_{n} & 0\end{array}\right], D_{d}=\left[\begin{array}{c}I_{p} \\ -K\end{array}\right], d$ is the disturbance on the plant states (i.e. the joint positions, velocities and integrators), $B_{n}=B_{d}, D_{d}=\left[\begin{array}{c}0 \\ -K\end{array}\right]$ and $m$ is the measurement noise on the plant output. Equations $(17 \& 18)$ are simulated in the next section to study the robustness of the control system.

\subsection{Relative stability}

The Nyquist diagram of determinant of the return difference at the input and output of the plant is utilized to serve as a rough estimate for relative stability. The return difference at the input and output of the plant are $F_{i}(s)=\left(I_{n}+K\left(s I_{p}-A\right)^{-1} B\right)$ and $F_{o}(s)=\left(I_{p}+\right.$ $\left.\left(s I_{p}-A\right)^{-1} B K\right)$ respectively. Nyquist diagram for the determinant of $F_{i}(s)$ is illustrated in the next section.

\section{SIMULATION RESULTS}

In this section, the linear model of C-Cub with 10 DoF is used to compare the centralized LQR and the decentralized PID in terms of time response, performance and robustness to noise and disturbances. The penalty matrices for the LQR design are:

$$
Q=\operatorname{diag}\left\{2500 \times I_{n}, \quad 1000 \times I_{n}, \quad I_{n}\right\}, \quad R=5 \times I_{n}
$$

Due to the ordering of the state space model the penalties are on joint angles, joint velocities and integrator states respectively. The LQR feedback gains result in an optimal cost of 607.6 while the initial gain for the iteration has a cost of 930.5 .

Remark 2: Regarding the initial stabilizing feedback gain that is used in the iterative scheme, it should be pointed out that for $\mathrm{C}$-Cub model the penalty on the input had to be reduced to derive the initial decentralized stabilizing controller $\left(K_{0}\right)$. This suggests that the LQR has to be of sufficient bandwidth in order to use the algorithm presented to synthesis the equivalent decentralized controller. Hence the iterative algorithm presented is only valid for a subset of the possible decentralized controllers.

The step size $\alpha$ is set to $5 \mathrm{e}-4$ and after 4000 iterations the cost of the decentralized PID is reduced to 653.7 which is $7.6 \%$ higher than the optimal cost, as shown in Fig. 1. The initial condition for the simulation is:

$$
q=\left[\begin{array}{llllllllll}
.1 & .2 & -.2 & -.1 & .2 & -.2 & .1 & .2 & -.2 & -.1
\end{array}\right]^{T}
$$

for the joint angles and zero vectors for the velocity and integrators initial conditions. The joint angles ordering used in this simulation is ankle lateral, ankle sagittal, knee, hip lateral, hip sagittal for the support leg (right leg) and mirror of this ordering is used for the swing leg (left leg). The C-Cub's initial posture corresponds to bending the ankles, knee and hips in the sagittal plane

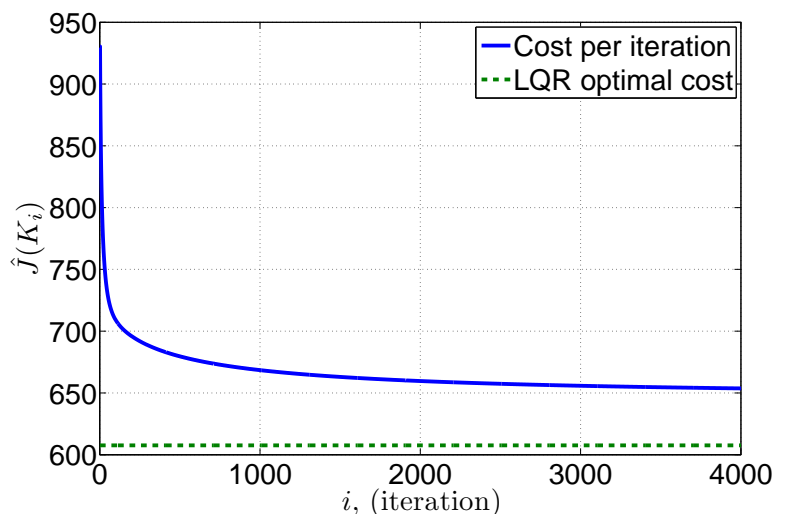

Fig. 1. Cost of the LQR objective function $\hat{J}\left(K_{i}\right)$ shown against the number of iterations.

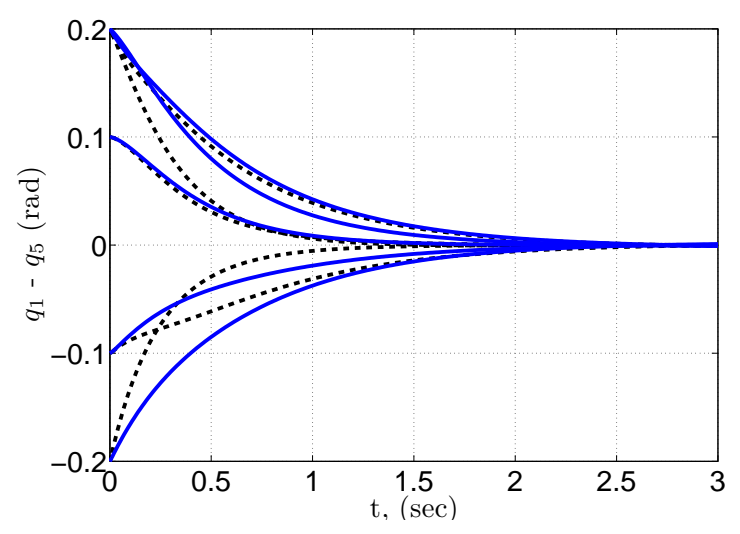

Fig. 2. C-Cub support leg joint positions' time response to the decentralized controller (dashed line) and the centralized controller (solid line).

for $0.2 \mathrm{rad}$ and a sway of $0.1 \mathrm{rad}$ in the lateral plane in such a way that the feet are kept parallel to the ground. The joint angles time response that regulates the angles to zero using the centralized and the decentralized controller is shown in Fig. $2 \& 3$ for the right and the left legs. The corresponding control signals (torques) generated by the two control schemes are illustrated in Fig. $4 \& 5$. The decentralized controller is clearly using higher torque compared to the centralized LQR controller. In particular in Fig. 4 the transient torque required by the decentralized controller is 5 to 6 times larger than the LQR signal. In a comparison between the stance leg joint angles and the corresponding torques it turns out that the decentralized controller is moving the knee and ankle in sagittal plane faster compared to the LQR that regulates all the joints with a more uniform trend. This effect can cause actuator saturation of knee and ankle joints. C-Cub can produce torques between 40-80 N-m depending on the joints' motor and gearbox reduction ratio. Hence the amount of torque shown in these figures can cause saturation during walking when joint angles larger than 0.2 or 0.1 radians is required.

In Fig. 6, the determinant of the return difference at the input of the plant is considered as a rough measure for relative stability of the feedback system. In Fig. 7, the maximum and minimum singular values of the closed loop 


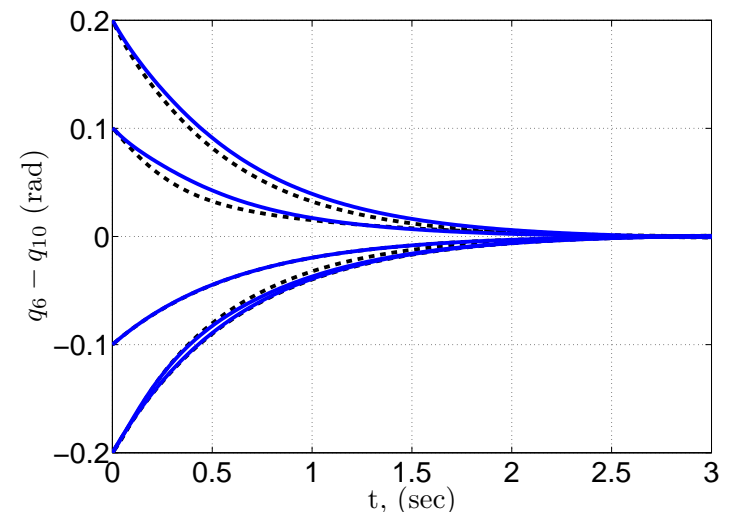

Fig. 3. C-Cub swing leg joint positions' time response to the decentralized controller (dashed line) and the centralized controller (solid line).

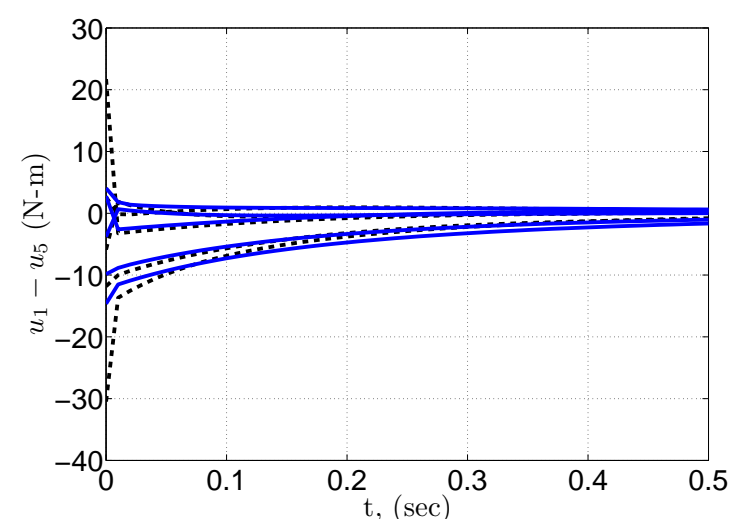

Fig. 4. C-Cub support leg joint torques required by the decentralized controller (dashed line) and the centralized controller (solid line).

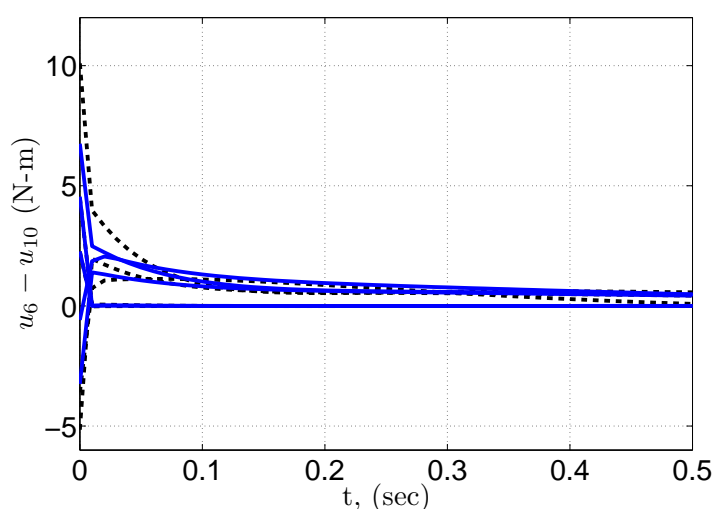

Fig. 5. C-Cub swing leg joint torques required by the decentralized controller (dashed line) and the centralized controller (solid line).

transfer function matrix from the reference input to joint positions is shown. The closed loop bandwidth is approximately $0.87 \mathrm{hz}$. In addition, the effect of disturbance and noise on the control system as given by Equations (17 \& 18) is simulated for the C-Cub model. In Fig. 8, a conservative measure of the maximum and minimum singular values of the disturbance rejection model on the

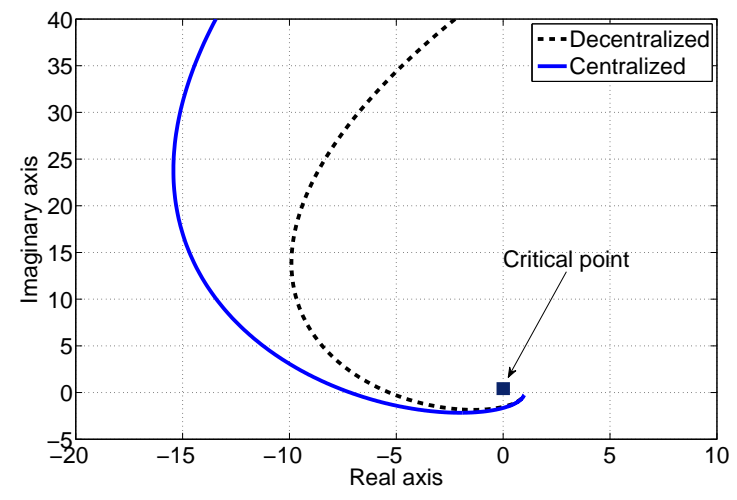

Fig. 6. Nyquist diagram of $F_{i}(s)$ to investigate the relative stability.

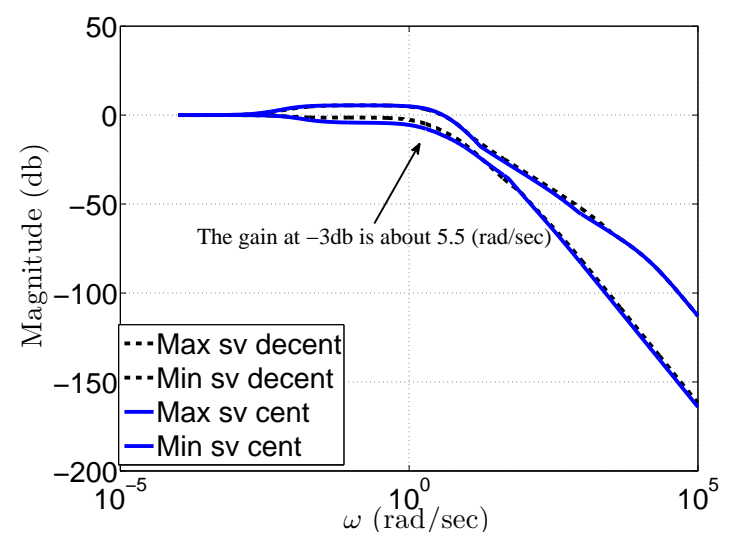

Fig. 7. Singular values of the closed loop transfer function matrix.

joint angles is illustrated. In this model the effect of disturbance injected at the output on the joint angles is shown. It can be seen that the centralized controller has better attenuation of such disturbances. In Fig. 9, the noise rejection performance of the closed loop system is shown. Although, at the frequency range of $1 \mathrm{e}-3$ to $1 \mathrm{e}-$ $1 \mathrm{rad} / \mathrm{sec}$ the noise on the the joint position gets amplified, in the higher frequencies where the noise is expected there is good attenuation. In addition the feedback has good attenuation properties for the noise on velocities and integrator states. See Fig. 10 for the joint velocities noise. A similar behaviour is observed for the integrators. In terms of performance under the same bandwidth the centralized control system provides more energy efficiency which is illustrated by the control signals required. Also the robustness of the centralized control system is better for disturbance and noise rejection.

\section{CONCLUSION}

This paper has presented a comparison between two centralized and decentralized control schemes for safe and robust control of walking in humanoid robots. Most advanced humanoid robots use a decentralized control system to stabilized the robot in different phases of walking (i.e. single support and double support) mainly due to their hardware architecture and simplicity of PID implementation, but little work has been published on the 


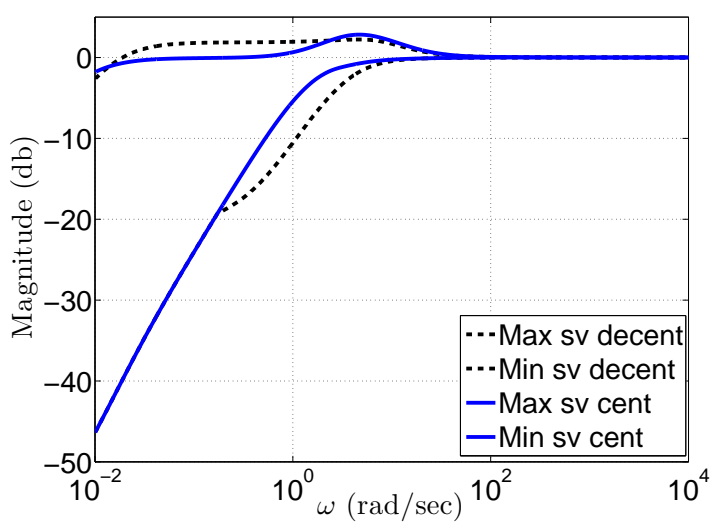

Fig. 8. Effect of disturbance on the joint angles. Singular values of disturbance model for the joint angles outputs.

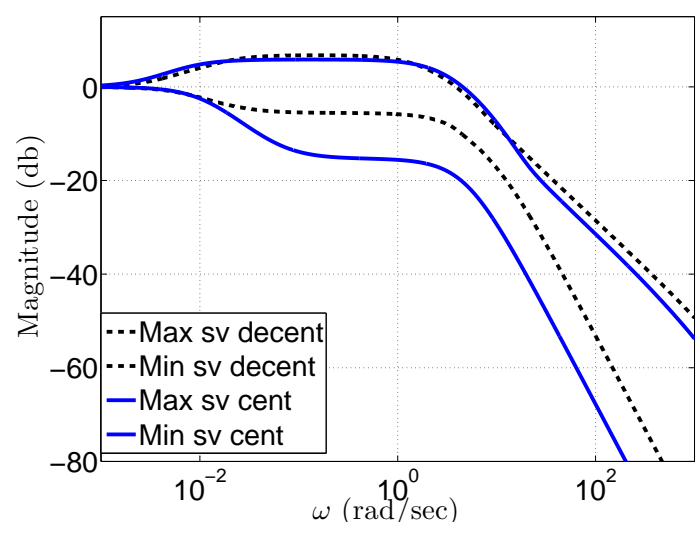

Fig. 9. Effect of quantization noise on the joint angles. Singular values of noise model for the joint angles outputs.

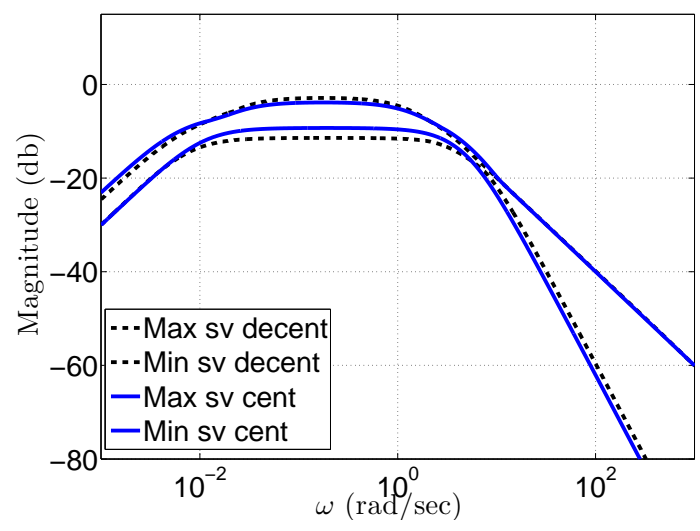

Fig. 10. Effect of velocities measurement noise on the joint angles. Singular values of noise model for the joint velocities outputs.

methods used for tuning such controllers. Given the multivariable model of the robot an iterative scheme was used to derive the decentralized PID controller from the centralized design. Both controllers were designed with the same bandwidth. The regulation performance, control signals disturbance and noise rejection of the two controllers were compared. Although the decentralized controller showed comparable properties in terms of regulation of joint angles and noise rejection, it requires relatively high control signals that can cause actuator saturation and instability of the humanoid see Fig. 4 \& 5. Furthermore, the centralized controller showed better disturbance rejection properties as can be seen in Fig. 8. This work, used a classical control method as a basis for quantification of robustness and performance of humanoid robots and it was shown that in general a better robustness is obtained from the centralized controller.

Future work will focus on modification of the iterative algorithm presented and inclusion of the drive dynamics to implement the controllers on the actual hardware. Also robust trajectory generation methods based on foot placement strategies will be investigated to provide a more robust control system for the humanoid walking.

\section{ACKNOWLEDGEMENTS}

The authors would like to thank the CICADA project and the Italian Institute of Technology, Genova, especially Profs. D. Caldwell, J. Gray and Dr. N. Tsagarakis for their support.

\section{REFERENCES}

Kaneko, K., Kanehiro, F., Kajita, S., Morisawa, M., Fujiwara, K., Harada, K. and Hirukawa, H. (2005). Slip observer for walking on a low friction floor. Proceedings of IEEE/RSJ International Conference on Intelligent Robots and Systems, 1(4), 634 - 640.

Kaneko, K., Kanehiro, F., Morisawa, M., Miura, K., Nakaoka, S. and Kajita, S. (2009). Cybernetic human HRP-4C. In Proceedings of the 9th IEEE-RAS International Conference on Humanoid Robots, 7-14.

Levine, W.S. and Athans, M. (1970). On the determination of the optimal constant output feedback gains for linear multivariable systems. IEEE Transactions on automatic control, 15(1), 44-48.

Medrano-Cerda, G.A., Dallali, H., Brown, M., Tsagarakis, N.G. and Caldwell, D.G. (2010). Modelling and simulation of the locomotion of humanoid robots. In $U K$ Automatic Control Conference, Coventry, 7-10 September.

Pratt, J., Carff,J., Drakunov,S. and Goswami,A. (2006). Capture point: A step toward humanoid push recovery. In Proceedings of the 6th IEEE-RAS International Conference on Humanoid Robots, 200-207.

Robotcub Project (2010). An open framework for research in embodied cognition. URL www.robotcub.org.

Silva, E.I. and Erraz, D.A. (2006). An LQR based MIMO PID controller synthesis method for unconstrained lagrangian mechanical systems. In Proceedings of the 45 th IEEE Conference on Decision and Control, 6593-6598.

Vukobratovic, M. and Borovac, B. (2004). Zero Moment Point - thirty five years of its life. Int. J. Humanoid Robotics, 1(1), 157-173.

Wight, D.L., Kubica, E.G. and Wang D.W.L. (2008). Introduction of the foot placement estimator: A dynamic measure of balance for bipedal robotics. J. Comput. Nonlinear Dynam, 3(1), 011009. 